\title{
Detection of ABO Discrepancy in a Case of Coronary Artery Disease by Conventional Tube Technique: A Miss by Column Agglutination Technology
}

\author{
Suman Sudha Routray ${ }^{1}$ Satya Prakash ${ }^{1}$ Gopal Krushna Ray ${ }^{1} \quad$ Somnath Mukherjee ${ }^{10} \quad$ Ansuman Sahu $^{1}$ (1) \\ ${ }^{1}$ Department of Transfusion Medicine, All India Institute of Medical \\ Sciences, Bhubaneswar, Odisha, India \\ Address for correspondence Ansuman Sahu, MD, Department of \\ Transfusion Medicine, All India Institute of Medical Sciences, \\ Bhubaneswar, 751019, Odisha, India (e-mail: ansu.vss@gmail.com).
}

J Lab Physicians 2022;14:87-89.

\begin{abstract}
Keywords

- $\mathrm{ABO}$

- blood group

- conventional tube technique

- column agglutination technique
\end{abstract}

\section{Introduction}

$\mathrm{ABO}$ and $\mathrm{Rh}$ blood grouping of the donors and recipient is a critical step in pretransfusion compatibility testing. Conventional tube technique (CTT) is the gold standard test for blood grouping. But it is a labor-intensive procedure with subjective variability in reporting of results. ${ }^{1}$ Moreover, it is not adaptable to automation. Many newer techniques such as the column agglutination technique (CAT) have overcome their limitations. Most blood centers are shifting to semi- automated or fully automated platforms. The CAT is used widely in these platforms for serologic testing because of increased sensitivity and stable end point results. ${ }^{2}$ This technique has been widely evaluated on automated platforms and equally efficient and reliable as CTT. However, it is sometimes observed that CAT misses subgroups detection. ${ }^{3}$ Here, we report a case with a subgroup of A that was failed to be identified by the CAT using dextran acrylamide gel, signifying the use of CTT in evaluating blood group discrepancy. published online January 18, 2022
DOI https://doi.org/ 10.1055/s-0041-1741440. ISSN $0974-2727$.
(C) 2022. The Indian Association of Laboratory Physicians. All rights reserved.

This is an open access article published by Thieme under the terms of the Creative Commons Attribution-NonDerivative-NonCommercial-License, permitting copying and reproduction so long as the original work is given appropriate credit. Contents may not be used for commercial purposes, or adapted, remixed, transformed or built upon. (https://creativecommons.org/ licenses/by-nc-nd/4.0/)

Thieme Medical and Scientific Publishers Pvt. Ltd., A-12, 2nd Floor, Sector 2, Noida-201301 UP, India 

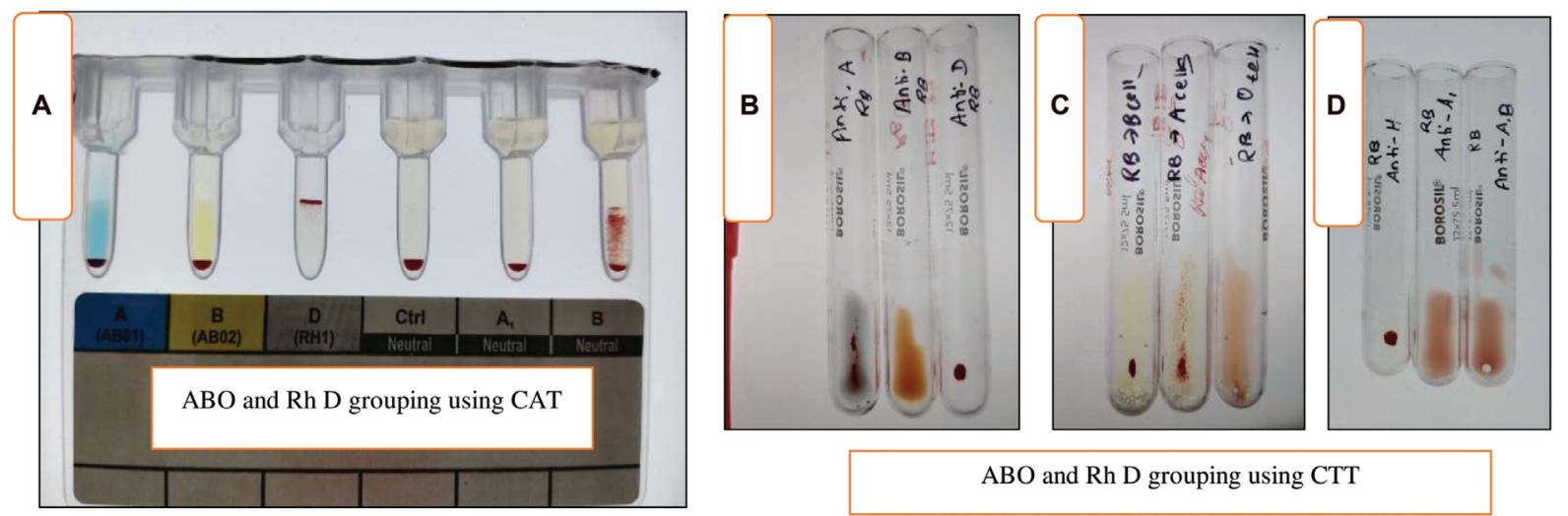

Fig. 1 ABO and Rh D grouping using column agglutination technique (CAT) (A) and conventional tube technique (CTT) (B-D).

\section{Case Report}

A blood sample of a male patient aged 65 years with coronary artery disease (CAD) was sent to the blood bank for ABO and Rh $D$ group typing. The patient did not have any previous history of blood transfusion. Forward and reverse blood grouping were performed using Matrix ABO/Rho (D) Forward and Reverse Grouping Card with autocontrol prefilled with a dextran acrylamide gel containing monoclonal anti-A (clone 11H5), anti-B (clone 6F9), anti-D (immunoglobulin M) (VI-) (clone P3 $\times 61+\mathrm{TH}-28$ ) from microtube 1 to 3 and neutral gel from microtube 4 to 6 (Tulip Diagnostics Pvt. Ltd., Goa, India, LOT No. 2004, expiry February 2021). For forward grouping, 5\% cell suspension was prepared using Matrix Diluent-2 LISS and $10 \mu \mathrm{L}$ of it was added to each of anti-A, anti-B, anti-D impregnated gel system. For reverse grouping, $50 \mu \mathrm{L}$ of pooled $A_{1}$ cells and $B$ cells ( $0.8 \%$ cell suspension, respectively) were added to the $A_{1}$ and $B$ labeled microtubes; $50 \mu \mathrm{L}$ of pooled $O$ cells $(0.8 \%$ cell suspension) was added to the control labeled microtube; $50 \mu \mathrm{L}$ of serum was added to each $A_{1}, B$, control microtubes, and incubated at room temperature for 10 minutes. The card was centrifuged for 10 minutes in the gel card centrifuge (CC1600, Matrix, Tulip Diagnostic), and the result was recorded. It showed a negative reaction with anti-A, anti-B, and a positive reaction (strength $4+$ ) with anti-D. A positive reaction was noted only with $B$ pooled cells (strength $4+$ ) (-Fig. 1A) on reverse grouping (-Table 1). Since blood group discrepancy was noticed, the test was repeated using CTT as per institutional protocol using anti-A, anti-B, and antiD ( - Fig. 1B) in the forward grouping and pooled $A_{1}, B$, and $O$ cells ( $\mathbf{- F i g . ~ 1 C )}$ in the reverse grouping. The red cells were further tested with anti-AB, anti- $\mathrm{A}_{1}$ lectin, and anti-H lectin (-Fig. 1D) to resolve the discrepancy. For forward grouping, 5\% cell suspension was added to three test tubes containing monoclonal antisera anti-A, anti-B, and anti-D (Tulip Diagnostic). For reverse grouping, two drops of serum were added to the test tubes containing one drop of $5 \%$ cell suspension of pooled $A_{1}, B$, and $O$ cells, respectively, and were incubated for 30 minutes at room temperature. Results were recorded after centrifuging the tubes (Table Top Centrifuge, Eppendorf, Germany) at $1,000 \mathrm{rpm}$ for 1 minute. For testing with anti$\mathrm{AB}$, anti- $\mathrm{A}_{1}$, and anti-H, $5 \%$ suspension of the red cell was used as forward grouping. Forward grouping revealed a positive reaction (strength + mixed field) with anti-A and anti-AB, while reverse grouping demonstrated a positive reaction with both $A_{1}$ (strength $3+$ ) and $B$ cells (strength $4+$ ). Anti- $A_{1}$ lectin exhibited no reaction, while $4+$ reaction was noted with anti-H lectin. Antibody screening using a commercial cell panel (ID-DiaCell I-II-III, Bio-Rad, LOT No. 45184.46.x, expiry December 14, 2020, Switzerland) was negative. Cold adsorption with gentle heat elution at $45^{\circ} \mathrm{C}$ confirmed A subgroup. Secretor study was not performed as the presence of "A antigen" in the red blood cells (RBCs) was evidenced by cell agglutination with anti-A antisera and anti$\mathrm{AB}$ antisera during testing in CTT as well as adsorption elution studies.

\section{Discussion}

We suspected a blood group discrepancy of either missing antigen or discrepancy due to unexpected ABO isoagglutinin

Table 1 Results of forward and reverse grouping using CAT and CTT

\begin{tabular}{|c|c|c|c|c|c|c|c|c|c|c|}
\hline \multirow[t]{2}{*}{ Technique } & \multirow[t]{2}{*}{ Anti-A } & \multirow[t]{2}{*}{ Anti-B } & \multirow[t]{2}{*}{ Anti-AB } & \multirow[t]{2}{*}{ Anti-D } & \multirow{2}{*}{$\begin{array}{l}\text { Anti- } A_{1} \\
\text { lectin }\end{array}$} & \multirow[t]{2}{*}{ Anti-H lectin } & \multicolumn{3}{|c|}{ Pooled cells } & \multirow[t]{2}{*}{ Inference } \\
\hline & & & & & & & $A_{1}$ & B & 0 & \\
\hline CAT & 0 & 0 & - & $4+$ & - & - & 0 & $4+$ & - & $\begin{array}{l}\text { Subgroup A } \\
\text { Group O with missing } \\
\text { antibody }\end{array}$ \\
\hline CTT & $+\mathrm{mf}$ & 0 & $+\mathrm{mf}$ & $4+$ & 0 & $4+$ & $3+$ & $4+$ & 0 & Subgroup of $A$ with anti- $A_{1}$ \\
\hline
\end{tabular}

Abbreviations: CAT, column agglutination technique; CTT, conventional tube technique; mf, mixed field (clumps of cells surrounded free cells). 
missed by CAT. But the routine CTT method detected the missing antigen without increasing the cell-serum ratio or incubation time. This case revealed the importance of CTT in evaluating blood group discrepancy and failure of CAT to identify the subgroup.

Blood group discrepancy exists when forward grouping does not corroborate with the reverse grouping. These are classically divided into four major types. Group I discrepancies are the most common type and result due to unexpected reactions in the reverse grouping, either missing or weakly reacting antibody(ies). Group II discrepancies are due to missing or weakly reacting antigen(s). Group IV discrepancy occurs due to miscellaneous causes such as unexpected $\mathrm{ABO}$ or non-ABO isoagglutinin. Resolution of groups II and IV discrepancy requires complex enhancing procedures. ${ }^{4}$ Here, we suspected group I or group II discrepancy based on the results of CAT. Repeat blood grouping with CTT revealed group II and group IV discrepancy in the form of a subgroup of $A$ with anti- $A_{1}$. Studies from different corners of the world reported $A_{3}$ being the most commonly encountered weaker subgroup of $A$ apart from $\mathrm{A}_{2}$ in the blood donor population. ${ }^{5,6}$ But the literature on the existence of weaker subgroups in the patient population is limited and mostly confined to identifying $A_{2}$ and $A_{2} B$ subgroups. Weak agglutination with anti-A suggests the possible presence of $A_{3}$ or $A_{x}$ phenotype. $A_{\text {end }}$ phenotype shows weak agglutination with anti-AB only. Anti$A_{1}$ is almost always present in $A_{x}$ whereas sometimes in $A_{3}$ and $A_{\text {end }}$ phenotypes. Complex adsorption elution studies are required to identify $A_{m}, A_{y}$, and $A_{e l}$ subgroup of $A$. Serological characteristics observed here suggest $A_{3}$ phenotype.

CAT involving microtubes either filled with gel or glass bead containing buffer and reagents is thoroughly evaluated for $\mathrm{RBC}$ antibody testing. These systems are found to be superior to CTT in the detection of clinically significant antibodies. ${ }^{7}$ Moreover, a gel-based system was better than glass bead-based technology in detecting weak antibodies in stored samples. ${ }^{8}$ But its use in blood typing is a relatively newer concept, gaining substantial interest only a while ago. Despite time consuming, CAT is used because of the simplicity of the process and reproducibility of results with stable end points. It is equally sensitive as CTT for ABO blood grouping and Rh D typing, but weak $\mathrm{A}$ or $\mathrm{B}$ phenotypes are better detected using CTT. ${ }^{9}$ It may be due to the reduction in the proportion of antigen compared with the antibody proportion to attend the zone of equivalence as the number of antigens is less on the weaker variants of A or B and only $0.8 \%$ red cell suspension is used in gel-based technology. Moreover, the absence of anti- $\mathrm{AB}$, anti- $\mathrm{H}$, and anti- $\mathrm{A}_{1}$ lectin in the microcolumns of the gel-based assay in a forward grouping may affect the interpretation of weaker variants.

Weaker variants of the ABO subtype are often mistyped as $O$ group due to variations in techniques and reagents. Accurate determination of subgroup variants in such cases helps in better management and predicting disease course. Individuals with blood group $\mathrm{O}$ have $67 \%$ decreased chances of developing venous thromboembolism than other non-O group individuals. Among non-O group individuals, subgroups of $A\left(A_{2}\right)$ have a lower risk than others because of decreased $\mathrm{H}$ antigen glycosylation resulting in an enzyme with reduced transferase activity. But these individuals have increased thrombotic risk as compared with $\mathrm{O}$ blood group individuals. Moreover, patients with A blood phenotype have an increased risk of myocardial infarction (MI). The alleles at rs514659 have been associated with CAD with MI through coronary thrombosis modulation and rupture of atherosclerotic plaque. ${ }^{10}$ Group 0 packed red blood cell and $A B$ plasma can be transfused to these types of the patient whenever indicated. Identification of subgroup in donors is also essential as it may be wrongly transfused to $O$ group recipient leading to decreased RBC survival.

\section{Conclusion}

Identification of weaker variants is of utmost importance from both donor and patient perspectives. Though CAT has a higher sensitivity to antigen-antibody reaction, it still missed the identification of weaker variants. CTT is the gold standard and should be used in evaluating discrepant cases. Genotyping in discrepancy cases with CAD can help in the prognostication of the disease.

\section{Funding \\ None.}

\section{Conflict of Interest}

The authors declare no conflicts of interest.

\section{References}

1 Rumsey DH, Ciesielski DJ. New protocols in serologic testing: a review of techniques to meet today's challenges. Immunohematology 2000;16(04):131-137

2 Dara RC, Tiwari AK, Mitra S, et al. Comparison of a column agglutination technology-based automated immunohematology analyzer and a semiautomated system in pretransfusion testing. Asian J Transfus Sci 2019;13(02):115-119

3 Shin SY, Kwon KC, Koo SH, et al. Evaluation of two automated instruments for pre-transfusion testing: AutoVue Innova and Techno TwinStation. Korean J Lab Med 2008;28(03): 214-220

4 Tiwari AK, Setya D, Arora D, Mehta SP, Aggarwal G, Mitra S. An algorithmic approach to serological work-up of $A B O$ sub-groups which present as $A B O$ discrepancies in resource constraint settings. J Immunol Methods 2020;487:112895

5 Thakral B, Saluja K, Bajpai M, Sharma RR, Marwaha N. Importance of weak ABO subgroups. Lab Med 2005;36(01):32-34. Doi: 10.1309/X59TAAYPEPCNBLUJ

6 Garretta M, Muller A, Gener J, Matte C, Moullec J. Reliability in automatic determination of the $\mathrm{ABO}$ group by the groupamatic system. Vox Sang 1974;27(02):141-155

7 Weisbach V, Ziener A, Zimmermann R, Glaser A, Zingsem J, Eckstein R. Comparison of the performance of four microtube column agglutination systems in the detection of red cell alloantibodies. Transfusion 1999;39(10):1045-1050

8 Sawierucha J, Posset M, Hähnel V, Johnson CL, Hutchinson JA, Ahrens N. Comparison of two column agglutination tests for red blood cell antibody testing. PLoS One 2018;13(12):e0210099

9 Langston MM, Procter JL, Cipolone KM, Stroncek DF. Evaluation of the gel system for ABO grouping and D typing. Transfusion 1999; 39(03):300-305

10 Zhang H, Mooney CJ, Reilly MP. ABO blood groups and cardiovascular diseases. Int J Vasc Med 2012;2012:641917 